\title{
Thiol-Functionalized Mesoporous Silica for Effective Trap of Mercury in Rats
}

\author{
Wei Zhao, ${ }^{1}$ Bo Song, ${ }^{2,3}$ Jie Tao, ${ }^{1}$ Jiumeng Zhang, ${ }^{1}$ Meijuan Huang, ${ }^{1,4}$ \\ Chun Wang, ${ }^{2}$ and Maling Gou ${ }^{1,4}$ \\ ${ }^{1}$ State Key Laboratory of Biotherapy and Cancer Center, West China Hospital, \\ Sichuan University and Collaborative Innovation Center for Biotherapy, Chengdu 610041, China \\ ${ }^{2}$ Chengdu Institute of Biology, Chinese Academy of Sciences, Chengdu 610041, China \\ ${ }^{3}$ College of Architecture and Environment, Sichuan University, Chengdu 610041, China \\ ${ }^{4}$ Department of Thoracic Oncology, Cancer Center, West China Hospital, Medical School, Sichuan University, Chengdu 610041, China
}

Correspondence should be addressed to Chun Wang; wangchun@cib.ac.cn and Maling Gou; goumaling@scu.edu.cn

Received 22 May 2016; Revised 31 July 2016; Accepted 24 August 2016

Academic Editor: Martin Andersson

Copyright (C) 2016 Wei Zhao et al. This is an open access article distributed under the Creative Commons Attribution License, which permits unrestricted use, distribution, and reproduction in any medium, provided the original work is properly cited.

\begin{abstract}
The chance of exposure to heavy metal for human being rises severely today due to the increasing water contamination and air pollution. Here, we prepared a series of thiol-functionalized mesoporous silica as oral formulation for the prevention and treatment of heavy metal poisoning. The successful incorporation of thiol was verified by the FTIR spectra. SBA15-SH-10 was used for the study as it is of uniform mesopores and fine water dispersibility. In simulated gastrointestinal fluid, the thiol-functionalized mesoporous silica can selectively capture heavy metal, showing a very high affinity for inorganic mercury (II). The blood and urine mercury levels of rats fed with a diet containing $\mathrm{Hg}$ (II) and material were significantly lower than those of rats fed with the metal-rich diet only. On the contrary, the mercury content in fecal excretion of the treatment group increased more than twice as much as that of the control group. This result indicated that SBA15-SH-10 could effectively remove mercury (II) in vivo and the mercury loaded on SBA15-SH-10 would be excreted out. Hence, SBA15-SH-10 has potential application in preventing and treating heavy metal poisoning via digestive system.
\end{abstract}

\section{Introduction}

The mesoporous silica has attracted great attention for its low cytotoxicity, excellent chemical stability, and rationally modifiable surface $[1,2]$. The large surface area and pore volume of mesoporous silica underlie facile adsorption and high loading effect of various therapeutic agents $[3,4]$. As a natural platform, the material has been used in pharmaceutics to improve drug formulation, drug bioavailability, and cellular targeting and to mitigate drug toxicity [5-7]. Furthermore, surface properties of mesoporous silica could be tailored, which allows us to tune the length scales and novel properties. These advantages indicate that mesoporous silica has potential application in toxin adsorption.

Heavy metal exposure becomes a growing health problem worldwide. Artisanal gold mining and the fossil fuels burning are the main causes for the release of mercury into the air and water $[8,9]$. Acute and high exposure to mercury occasionally happens among children by accidently swallowing mercury battery or liquid containing mercury (II) [10]. However, there is no efficient treatment before the hospitalization. There is possibility of mercury remnant even receiving the treatment of gastrolavage and clyster. Rationally designed nanoparticles capable of binding toxins show potential application in detoxification [11, 12]. By taking advantages of the large surface area of the mesoporous silica materials, we intend to design an oral antidote for metallic cations detoxification in the abovementioned cases. Nevertheless, the unmodified mesoporous silica cannot trap heavy metal efficiently.

In clinics, chelating agents like dimercaptosuccinic acid (DMSA, Succimer) are most often used to treat heavy metal intoxications, of which thiol is the functional group responsible for chelating heavy metals $[13,14]$. Inspired by the chelating agents, thiol group was employed for modifying 




FIgURE 1: Schematic demonstration of the detoxification strategy. Here, we prepared thiol-functionalized mesoporous silica as an oral formulation for the prevention and treatment of heavy metal poisoning. In simulated gastrointestinal fluid, thiol-functionalized mesoporous silica can selectively capture heavy metal, showing a very high affinity for inorganic mercury (II). In vivo, the material can significantly decrease the blood and urine mercury levels of the acute mercury poisoning rats.

the mesoporous nanoparticles [15]. The functionalized mesoporous materials exhibit great improvement in adsorption effectiveness and prefer to capture heavy metal ions. We attribute the effectiveness of such adsorbents to the complexation of grafted ligands with metal ions [16, 17]. Furthermore, the adsorbents will not be taken up by cell or damage the cell membrane when the particle size is greater than $5 \mu \mathrm{m}$ [18]. In this work, SBA-15 was used as the framework considering the highly ordered (four- to seven-peak XRD pattern) twodimensional $(2 \mathrm{D})$ hexagonal $(p 6 \mathrm{~mm})$ mesostructure and thick uniform silica walls (31 to $64 \AA$ ), which may impart significant stability to SBA-15 in comparison to MCM-41 [19].

In this work, we demonstrated that the thiol-functionalized mesoporous silica (SBA15-SH-10) could selectively trap heavy metal ions. Then, we constructed an acute mercury (II) poisoning model on rats and treated them with SBA15SH-10 (Figure 1). Ten minutes after mercury (II) is given, the SBA15-SH-10 was administrated orally, which significantly decreased the mercury levels of blood and urine. Altogether, the accessible thiol-functionalized SBA-15 type hybrid mesoporous silica can efficiently trap mercury in vitro and in vivo and has potential application in heavy metal detoxification.

\section{Materials and Methods}

2.1. Materials. Triblock copolymer pluronic 123 (P123, $\mathrm{EO}_{20} \mathrm{PO}_{70} \mathrm{EO}_{20}, M_{\mathrm{av}}=5800$ ) was purchased from Sigma
Chemical Co., St. Louis, USA. 3-Mercaptopropyltrimethoxysilane (MPTES) and tetraethyl orthosilicate (TEOS) were purchased from Tokyo Chemical Industry, Japan, and J\&K Scientific Ltd., China, respectively. Mercury (II) chloride, magnesium (II) chloride, and zinc (II) chloride were obtained from Aladdin Industrial Co., Shanghai, China. All used chemicals were of analytical grade, and all solvents and reagents were directly used without further purification.

2.2. Animals Procedure. 9-10 week-old male Sprague Dawley (SD) rats about 200 grams were obtained from Sichuan University laboratory animal center (Chengdu, Sichuan, China) and maintained under specific pathogen-free conditions with abundant food and water. Rats had seven days at least to adapt themselves to environment prior to experiments. All animal procedures were carried out according to the guidelines of the Animal Ethics Committee of Sichuan University (ethical approval number: 2015-0737).

2.3. Adsorbent Synthesis. The thiol-functionalized SBA-15 was synthesized as in the following procedure (Figure 1). Briefly, the molar ratio of MTPES to (MTPES + TEOS) in feed was kept at 0.1 . A total of $3 \mathrm{~g}$ of pluronic 123 that was used as structure directing agent was dissolved in $109 \mathrm{~mL}$ of $1.65 \mathrm{M} \mathrm{HCl}$ at room temperature. After the solution had been heated to $40^{\circ} \mathrm{C}, 0.037 \mathrm{~mol}$ of TEOS (employed as silica precursor) was added dropwise. Then, the resultant mixture 
was stirred for 45 minutes before the addition of $0.0041 \mathrm{~mol}$ of MPTES (used to provide the thiol groups). Following vigorously stirring for 24 hours at $40^{\circ} \mathrm{C}$, the reactant mixture was finally aged for 30 hours at $100^{\circ} \mathrm{C}$. After being cooled down to room temperature, the resultant particles were isolated by filtration and rinsed with water. P123 was extracted by Soxhlet extraction with ethanol for 24 hours. The material after P123 removing was denoted as SBA15-SH-10.

Other mesoporous materials with different degrees of thiol incorporation were prepared by similar method. The resultant materials were named as SBA15-SH-0, SBA15-SH5, SBA15-SH-20, and SBA15-SH-30, respectively.

2.4. Adsorbent Characteristics. Fourier transform infrared (FTIR) spectra were recorded using Perkin-Elmer system 2000 FT-IR spectrometer. Morphologic characteristic of the materials was determined by scanning electron microscopy (SEM) (JEOL 6300-F) and transmission electron microscope (TEM) (Tecnai-G2-F20). A quantitative analysis of the thiol group content was completed by the CHS elemental analysis (EA) (Perkin-Elmen 240C elemental analyzer). After the sample had been degassed at $150^{\circ} \mathrm{C}$ for 2 hours, nitrogen adsorption-desorption isotherms were measured at $77 \mathrm{~K}$ using a HYA2010-B2 system. The Barret-Joyner-Halenda $(\mathrm{BJH})$ pore size distribution was drawn from the desorption branch of the isotherm. The multiple-point Brunauer-Emmett-Teller (BET) specific surface area was computed in the relative pressure range $\left(P / P_{0}=0.05-0.3\right)$. Small-angle $\mathrm{X}$-ray diffraction (SXRD) measurement was performed on PANalytical X'Pert ${ }^{3}$ Powder instrument equipped with $\mathrm{Cu} \mathrm{Ka}$ $\mathrm{X}$-ray source of $\lambda=1.5406 \AA$ at $40 \mathrm{kV}$ and $40 \mathrm{~mA}$.

2.5. Heavy Metal Binding Capacity of Adsorbents. Before adsorption experiments, we measured the water dispersibility of materials by resolving $30 \mathrm{mg}$ material in $1 \mathrm{~mL}$ water. Batches of different experiments were carried out to test the adsorption ability of the materials under a series of $\mathrm{pH}$ conditions ranging from $\mathrm{pH}=1.2$ (simulated gastric fluid) to $\mathrm{pH}=7.0$ (simulated intestinal fluid). Briefly, a total volume of $10 \mathrm{~mL}$ aqueous solution of mercury (II) $(500 \mathrm{mg} / \mathrm{L})$ was stirred at $20^{\circ} \mathrm{C}$ for 24 hours in the presence of $20 \mathrm{mg}$ SBA15$\mathrm{SH}-10$. Then, the mixture was filtered with a $0.45 \mu \mathrm{m}$ syringe filter to collect the filtrate for the determination of mercury concentration using inductively coupled plasma emission optical spectrograph (ICP-OES) (SPECTRO ARCOS apparatus). Two emission mercury lines (184 and $193 \mathrm{~nm}$ ) were used for analysis. The mercury capture capacity of the material was determined by the difference between the initial and final concentration of mercury of the solution. The competitive adsorption ability of the material to different metal ions was measured at $\mathrm{pH}=5.5$ by the method mentioned above. The selected aqueous system was a mixture of $\mathrm{Hg}$ (II), Ca (II), and $\mathrm{Mg}$ (II). By the same way, mercury adsorption isotherms of the other thiol-functionalized materials were measured through several experimental runs with the initial mercury concentration ranging from 20 to $1800 \mathrm{mg} / \mathrm{L}$ at $20^{\circ} \mathrm{C}$ under the condition of simulated gastric fluid $(\mathrm{pH}=1.2)$.
2.6. Animal Experiments. In the experiments, SBA-SH-10 was used to test the detoxification ability of mercury in vivo. The rats were divided into two groups (control and treatment group) after each rat had been given $200 \mu \mathrm{L} \mathrm{HgCl}_{2}$ solution $(1 \mathrm{mg} / \mathrm{mL})$ through intragastric administration. Ten minutes later, the rats of treatment group received $1 \mathrm{mg}$ SBA15-SH10 in $800 \mu \mathrm{L}$ water, while rats of control group received $800 \mu \mathrm{L}$ water only. Animals were then housed individually in metabolic cages.

All the urine and faeces were collected throughout the experiment. At the end, a $2 \mathrm{~mL}$ sample of urine per rat was stored in a tube for the determination of mercury level. All the faeces discharged were counted for estimation of mercury content.

Forty-eight hours later, animals were anesthetized with chloral hydrate. All the blood samples were harvested from the abdominal aorta and stored in $15 \mathrm{~mL}$ BD tube (Becton, Dickinson and Company) for plasma separation. Then, $2 \mathrm{~mL}$ of plasma was set aside for the determination of mercury level.

2.7. Evaluation of Mercury Level. The mercury (II) in the sample of faeces was desorbed in the presence of proper amount of nitric acid $(5 \mathrm{~mol} / \mathrm{L})$. Wet digestion method with nitric acid, hydrogen peroxide, was used to treat the samples (plasma, urine, and desorbing solution of faeces) using Microwave Digestion System (MDS, APL Instrument Co., Ltd., China) for 90 minutes. Then, the mercury level of each sample was determined by cold vapour atomic absorption spectrophotometer (Spectra-AA 200FS, Varian).

2.8. Statistical Analysis. All the experiments were replicated three times. The data were shown as the mean \pm standard deviation and evaluated using Student's $t$-test or compared by one-way analysis of variance. $P<0.05$ was considered as significant.

\section{Results and Discussion}

Here, we prepared thiol-functionalized mesoporous silica as an oral formulation for the prevention and treatment of heavy metal poisoning (Figure 1). In simulated gastrointestinal fluid, thiol-functionalized mesoporous silica can selectively capture heavy metal, showing a very high affinity for inorganic mercury (II). In vivo, the material can significantly decrease the blood and urine mercury levels of the acute mercury poisoning rats. Hence, thiol-functionalized mesoporous silica has potential application in preventing and treating heavy metal poisoning via digestive system.

3.1. Adsorbent Preparation and Characterization. Highly ordered thiol-functionalized mesoporous silica was synthesized via cocondensation method. MPTES was employed as functional monomer, TEOS as silica source, and triblock copolymer as a template. All materials were synthesized by adding MPTES after $45 \mathrm{~min}$ of prehydrolysis of TEOS to allow the aromatic groups to fully interact with the surfactant micelles. The surfactant was removed by ethanol extraction. 


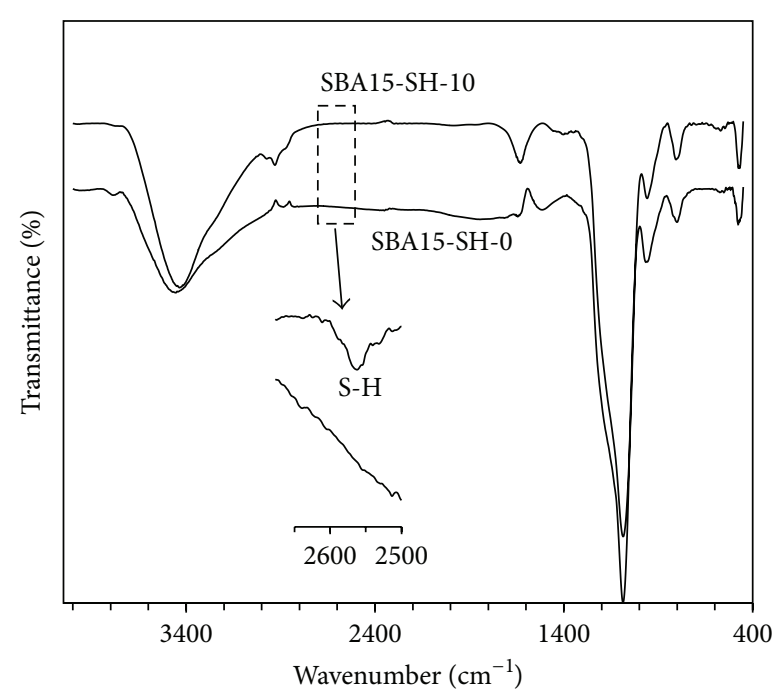

(a)



(c)

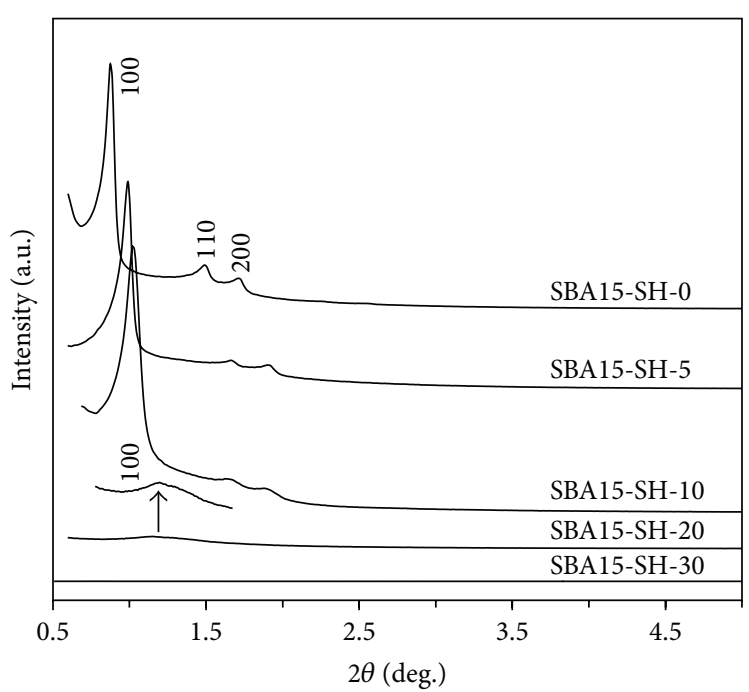

(b)

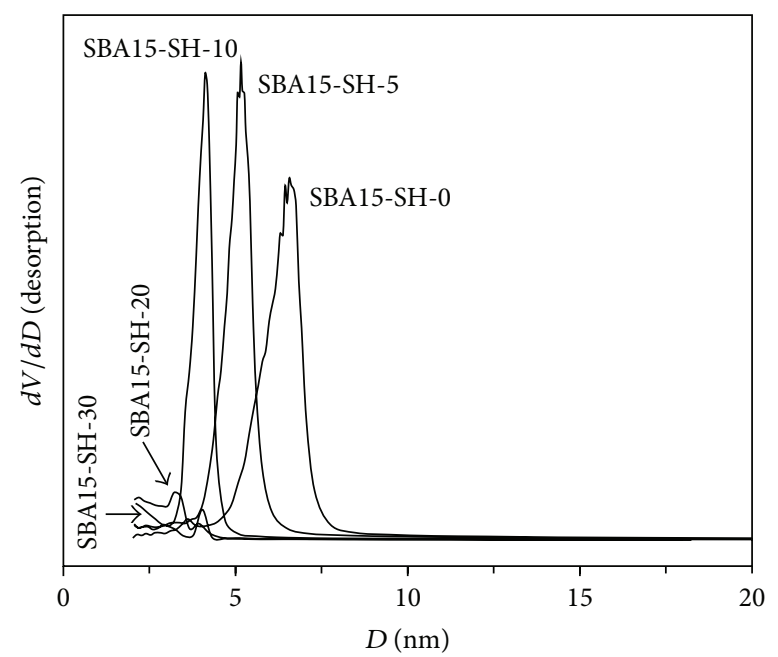

(d)

FIGURE 2: Characterization of the thiol-functionalized mesoporous silica. (a) FTIR image; (b) SXRD image; (c) $\mathrm{N}_{2}$ adsorption-desorption isotherms; (d) BJH pore size distribution curves.

The materials were then dried at $60^{\circ} \mathrm{C}$ for 24 hours for further analysis.

The FTIR spectra of SBA15-SH-0 and SBA15-SH-10 are shown in Figure 2(a). The bands at $2850-2900 \mathrm{~cm}^{-1}$ and $1449 \mathrm{~cm}^{-1}$ of the materials could be attributed to $\mathrm{C}-\mathrm{H}$ stretching and deformation vibrations [20]. Furthermore, SBA15-SH-10 has a weak vibrational band at $2600-2540 \mathrm{~cm}^{-1}$ assigning to the $\mathrm{S}-\mathrm{H}$ stretching vibration, while this band is blank for SBA15-SH-0. This qualitative evidence from FTIR spectra indicates that the thiol groups were successfully incorporated into the materials.

To further confirm the incorporation of mercaptopropyl groups, the sulfur content of the materials was analyzed by elemental analysis. The results are summarized in Table 1. Sulfur content of the materials increases from $0.59 \mathrm{mmol} \cdot \mathrm{g}^{-1}$ (SBA15-SH-5) to $3.01 \mathrm{mmol} \cdot \mathrm{g}^{-1}$ (SBA15-SH-30). The percentages of functional monomer (used and integrated) indicate that 57.6\% (SBA15-SH-5) to 95.7\% (SBA15-SH-30) of the added functional monomers are integrated into thiolfunctionalized mesoporous silica.

The SXRD was performed on all the materials and the results are shown in Figure 2(b). The SXRD patterns of SBA15-SH-0, SBA15-SH-5, and SBA15-SH-10 present a strong peak at $d(100)$ and two weak peaks at $d(110)$ and $d(200)$, which are characteristic of a $2 \mathrm{D}$ hexagonally ordered $(p 6 \mathrm{~mm})$ structure $[21,22]$. However, when the thiol content was greater than $20 \%$, the resultant materials were disordered, which could be attributed to the intense disturbance of too many organic precursors by the ionic interaction between the silicate species and the surfactant hydrophilic head groups [23].

Figure 2(c) shows the $\mathrm{N}_{2}$ adsorption-desorption isotherms of the materials at $77 \mathrm{~K}$. For SBA15-SH-10, the characteristic mesoporous type IV curve [24] is found possessing 




(a)

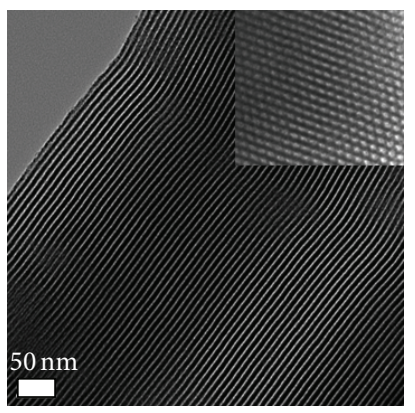

(e)

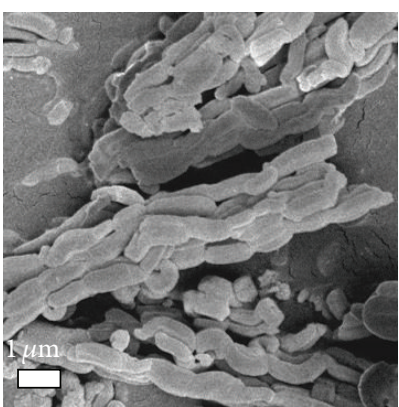

(b)

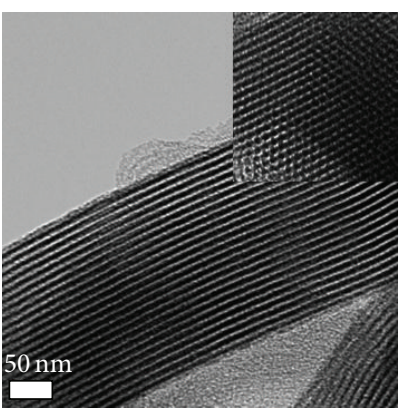

(f)

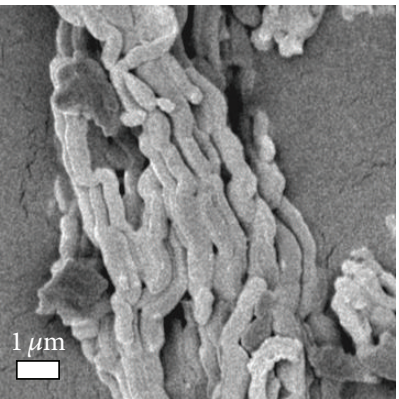

(c)

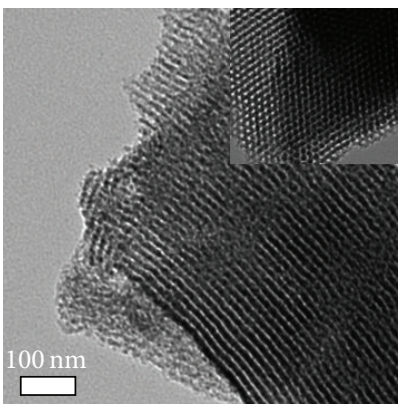

(g)

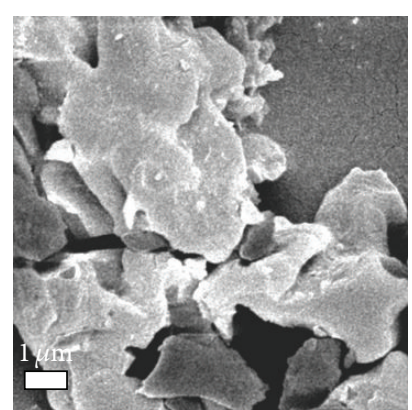

(d)

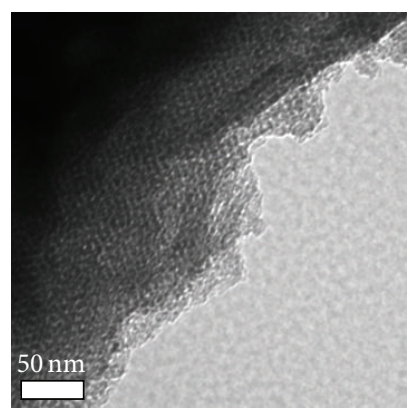

(h)

FIGURE 3: Morphological features of the materials determined by SEM and TEM. (a), (b), (c), and (d) were the SEM images of SBA15-SH-0, SBA15-SH-5, SBA15-SH-10, and SBA15-SH-20, respectively; (e), (f), (g), and (h) were the TEM images viewed from the pore axis and the perpendicular direction (inner graph) of SBA15-SH-0, SBA15-SH-5, SBA15-SH-10, and SBA15-SH-20, respectively.

TABLE 1: Incorporated thiol contents calculated from elementary analysis.

\begin{tabular}{|c|c|c|c|c|}
\hline \multirow[t]{2}{*}{ Materials } & \multirow[t]{2}{*}{ S content $(\%)$} & \multirow[t]{2}{*}{ Amount of thiol group $\left(\mathrm{mmol} \cdot \mathrm{g}^{-1}\right)$} & \multicolumn{2}{|c|}{$\begin{array}{l}\text { Percentage of functional } \\
\text { precursor }\end{array}$} \\
\hline & & & Used $^{\mathrm{a}}$ & Integrated $^{\mathrm{b}}$ \\
\hline SBA15-SH-0 & - & - & - & - \\
\hline SBA15-SH-5 & $1.88 \%$ & 0.59 & $5 \%$ & $2.9 \%$ \\
\hline SBA15-SH-10 & $3.03 \%$ & 0.94 & $10 \%$ & $5.1 \%$ \\
\hline SBA15-SH-20 & $7.20 \%$ & 2.25 & $20 \%$ & $17.1 \%$ \\
\hline SBA15-SH-30 & $9.65 \%$ & 3.01 & $30 \%$ & $28.7 \%$ \\
\hline
\end{tabular}

${ }^{a}$ Molar ratio of MPTES/(TEOS + MPTES) as added. ${ }^{b}$ Molar ratio of $-\mathrm{SH} / \mathrm{SiO}_{2}$ calculated using the $\mathrm{S}$ content from elemental analysis.

a sharp capillary condensation step at relative pressure $\left(P / P_{0}\right)$ of $0.45-0.65$, suggesting a very narrow pore size distribution. The hysteresis loop is of a H1 type [25], implying a uniform cylindrical pore geometry. SBA15-SH-0 and SBA15-SH-5 have the similar mesoporous types and hysteresis loops to SBA15-SH-10. The Brunauer-Emmett-Teller (BET) surface area, pore size, and pore volume are listed in Table 2, and the pore size distributions are displayed in Figure 2(d). When the molar ratio of MTPES to (MTPES + TEOS) is increased to $20 \%$, pore channels become slit shaped. The surface area and pore size of the materials decrease with the increment of thiol content. This is probably because the thiol groups can intensely interact with the hydrophobichydrophilic palisade region of the surfactant micelles, which could destroy the cooperation between silicon hydroxyls and surfactant micelles.
SEM images of the materials are shown in Figures 3(a)3(d). For SBA15-SH-0, SBA15-SH-5, and SBA15-SH-10, large fibrous structures with length of $10-20 \mu \mathrm{m}$ and diameter of 4-6 $\mu \mathrm{m}$ are observed. This fibrous structure is constituted of many rope-like subparticles with relatively uniform size, $1-2 \mu \mathrm{m}$ in length and $0.5 \mu \mathrm{m}$ in diameter. Nevertheless, the microstructure of SBA15-SH-20 becomes an irregular bulk. The mesoscopic ordering and symmetry deduced from the SXRD data are further confirmed by TEM (Figures 3(e)$3(\mathrm{~h})$ ). When the molar ratio of MTPES to (MTPES + TEOS) is below $10 \%$, these materials show excellent hexagonal ordering of mesopores in a long-range pore channel. On the contrary, the hexagonal ordering disappeared and the pore size becomes very small for SBA15-SH-20. Both SEM and TEM results indicate excess MPTES addition will destroy the microtacticity of the material. 
TABLE 2: Mercury adsorption performance parameters of the materials.

\begin{tabular}{|c|c|c|c|c|c|c|}
\hline Materials & $\begin{array}{l}\text { Pore size } \\
\quad(\mathrm{nm})\end{array}$ & $\begin{array}{c}S_{\mathrm{BET}} \\
\left(\mathrm{m}^{2} \mathrm{~g}^{-1}\right)\end{array}$ & $V_{p}\left(\mathrm{~cm}^{3} \mathrm{~g}^{-1}\right)$ & $\begin{array}{c}\text { Maximum } \\
\text { mercury loading } \\
\left(\mathrm{mmol} \mathrm{g}^{-1}\right)\end{array}$ & $\begin{array}{l}\text { Amount of thiol } \\
\text { group }\left(\mathrm{mmol} \cdot \mathrm{g}^{-1}\right)\end{array}$ & $\mathrm{Hg}-\mathrm{S}$ molar ratio \\
\hline SBA15-SH-0 & 6.56 & 689 & 1.14 & $0.09 \pm 0.01$ & - & - \\
\hline SBA15-SH-5 & 5.4 & 635 & 0.98 & $0.53 \pm 0.01$ & 0.59 & 0.90 \\
\hline SBA15-SH-10 & 4.3 & 529 & 0.65 & $0.94 \pm 0.05$ & 0.95 & 0.98 \\
\hline SBA15-SH-20 & 3.5 & 316 & 0.25 & $1.98 \pm 0.04$ & 2.25 & 0.88 \\
\hline SBA15-SH-30 & 3.0 & 280 & 0.17 & $2.59 \pm 0.05$ & 3.01 & 0.86 \\
\hline
\end{tabular}

3.2. In Vitro Evaluation of Adsorbent. Mercury adsorption isotherms of all materials are obtained at $20^{\circ} \mathrm{C}$ and $\mathrm{pH}=$ 1.2 (Figure 4(a)), where the amount of mercury adsorbed at equilibrium, $q_{e}$ (millimole per gram of adsorbent), is plotted as a function of the equilibrium mercury concentration in the liquid phase, $C_{e}$ (milligrams per liter). The maximum adsorption volume of mercury $\left(Q_{0}\right)$ was calculated from the linearized Langmuir equation:

$$
\frac{C_{e}}{q_{e}}=\frac{1}{Q_{0} b}+\frac{C_{e}}{Q_{0}},
$$

where $Q_{0}$ and $b$ are the characteristic Langmuir parameters related to the maximum adsorption capacity and the intensity of adsorption, respectively. The maximum adsorption capacity of all materials is summarized in Table 2. From the data, we can obtain that the maximum $\mathrm{Hg}^{2+}$ binding capacity of different materials increases with rising thiol content. The thiol groups of SBA15-SH-5 and SBA15-SH-10 show a high accessibility of mercury species with a near $1: 1 \mathrm{Hg}-\mathrm{S}$ stoichiometric bonding, while SBA15-SH-0 has a negligible $\mathrm{Hg}^{2+}$ binding capacity. This is probably because a majority of the thiol groups are located on the outer wall surface and inner pore surface of the materials which have a big ordering pore channel in favor of the cooperation between thiol groups and mercury species [23]. On the contrary, when the molar ratio of MTPES to (MTPES + TEOS) is more than $20 \%$, the channel becomes disordered and the pore size becomes smaller, which obstruct the incorporation between thiol groups and mercury species. The possible reason is that a mass of thiol groups run into the silica wall leading to the disorder of structure [26]. Furthermore, the isotherms are well fitted by a Langmuir model (Figure 4(b)). Simultaneously, the hydrophily of the materials was studied and the result is shown in Figure 4(e). The results indicate that SBA15-SH-20 and SBA15-SH-30 have a poor hydrophily, while the other materials possess outstanding water dispersibility. We think high thiol content and small pore size should take responsibility for the poor water dispersibility of the materials.

Theoretically, as the number of thiol groups per organosilane molecule increases, the adsorption capacity will increase too. However, high-loaded functional monomer is actually filling up the pores as well as covering the outer surface. Sanz et al. also reported that the amino functional monomer would block the pores of SBA15 as its content increased [26]. And the high-content thiol will destroy the microtacticity of the material and significantly reduce the water dispersibility
TABLE 3: Mercury levels detected in plasma, urine, and faeces.

\begin{tabular}{lccc}
\hline Samples & Control & Treatment & $P$ value \\
\hline Plasma $(\mu \mathrm{g} / \mathrm{L})$ & $67.83 \pm 5.53$ & $21.72 \pm 3.68$ & 0.0001 \\
Urine $(\mu \mathrm{g} / \mathrm{L})$ & $152.08 \pm 17.51$ & $23.19 \pm 5.75$ & 0.0001 \\
Faeces $(\mu \mathrm{g})$ & $9.54 \pm 0.35$ & $24.52 \pm 2.64$ & 0.0003 \\
\hline
\end{tabular}

of the material when the thiol content reaches $20 \%$, which will eventually affect material's heavy metal capture ability as well. Hence, considering the uniformity of the mesopores, adsorption capacity, and dispersibility, mesoporous silica with ten percentages of thiol incorporation (SBA15-SH-10) was used for the subsequent experiments.

We further investigated the influence of $\mathrm{pH}$ on the mercury (II) removal capacity and the experiment was conducted under specific $\mathrm{pH}$ value ranging from 1.2 to 7.0. Figure 4(c) shows that the mercury (II) adsorption capacity of SBA15SH-10 is between 0.91 and $1.08 \mathrm{mmol} / \mathrm{g}$, suggesting that the binding capacity is not significantly affected by $\mathrm{pH}$ within the tested range.

Competitive adsorption ability of SBA15-SH-10 to different metal ions were also tested in the presence of $\mathrm{Hg}$ (II), Ca (II), and Mg (II) in aqueous systems. The material functionalized by thiol exhibits notable withdrawal abilities for $\mathrm{Hg}$ (II) as shown in Figure 4(d). The mercury concentration of aqueous system went down to $20.5 \mathrm{mg} / \mathrm{L}$ from $302 \mathrm{mg} / \mathrm{L}$ suggesting that the $\mathrm{Hg}$ (II) adsorption ratio is around $93.2 \%$. However, the adsorbent exhibited weak or no affinity for $\mathrm{Ca}$ (II) and $\mathrm{Mg}$ (II) as the concentration changes were insignificant. This indicates that the SBA15-SH-10 holds selective capture ability for heavy metal.

3.3. Detoxification Effect of Adsorbent in Rats. To test the ability of detoxification of SBA15-SH-10 in vivo, samples of urine, faeces, and blood of rats were collected for mercury analysis. The analysis results of samples for evaluation of mercury level are summarized in Table 3 and Figure 5. The blood burden of mercury (II) in control group is $67.83 \pm 5.53 \mu \mathrm{g} / \mathrm{L}$, which is much greater than that in the treatment group $(P=0.0001)$. Besides, a notably increased urine mercury level of control group is observed, compared to that of treatment group $(P=$ $0.0001)$. On the contrary, the cumulative mercury content of faeces in treatment group increased more than twice as much as that in control group $(P=0.0003)$. This data demonstrates that the mercury volume accessed to the blood is very low 


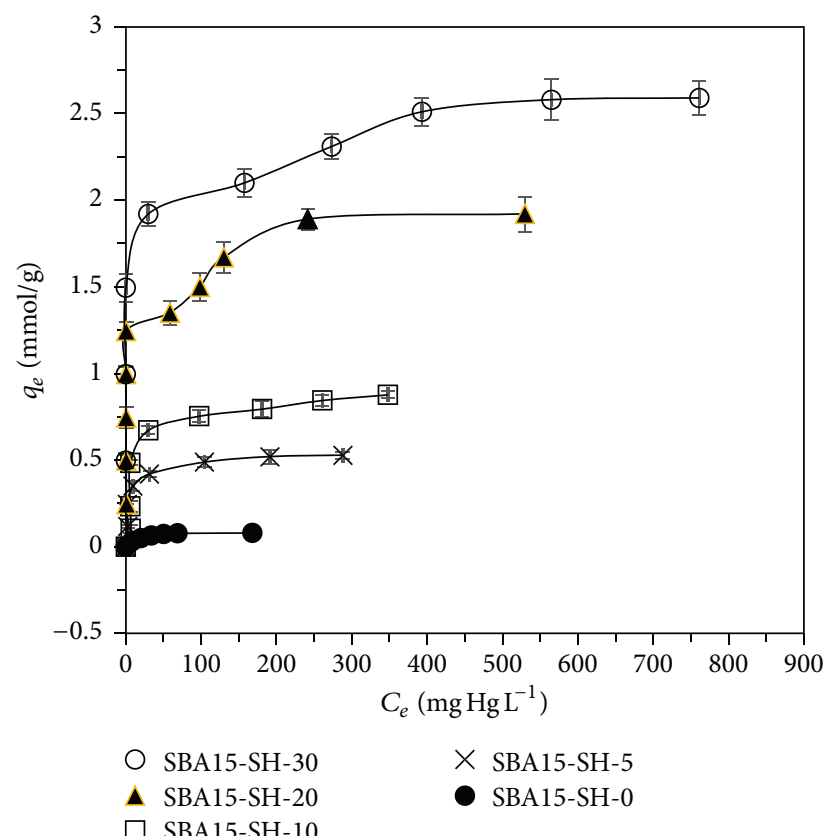

(a)

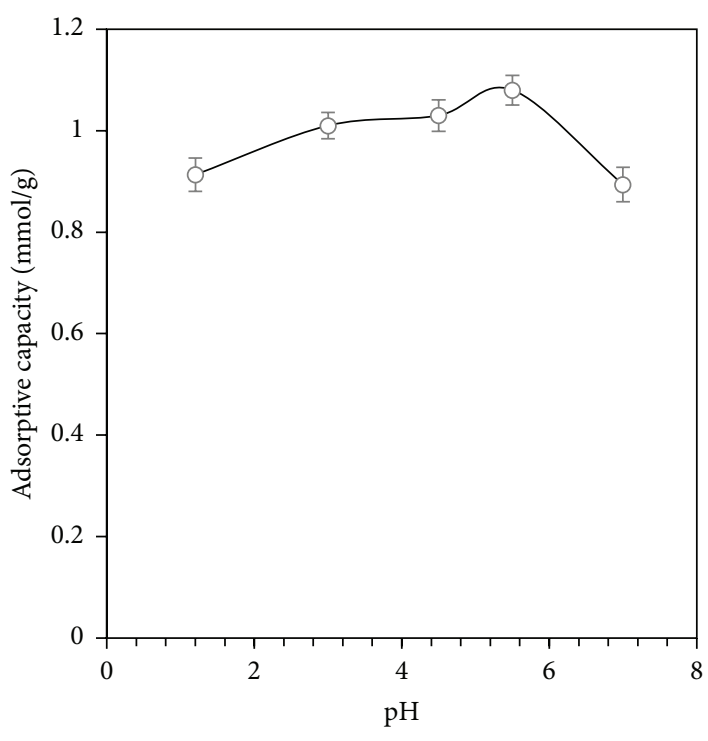

(c)

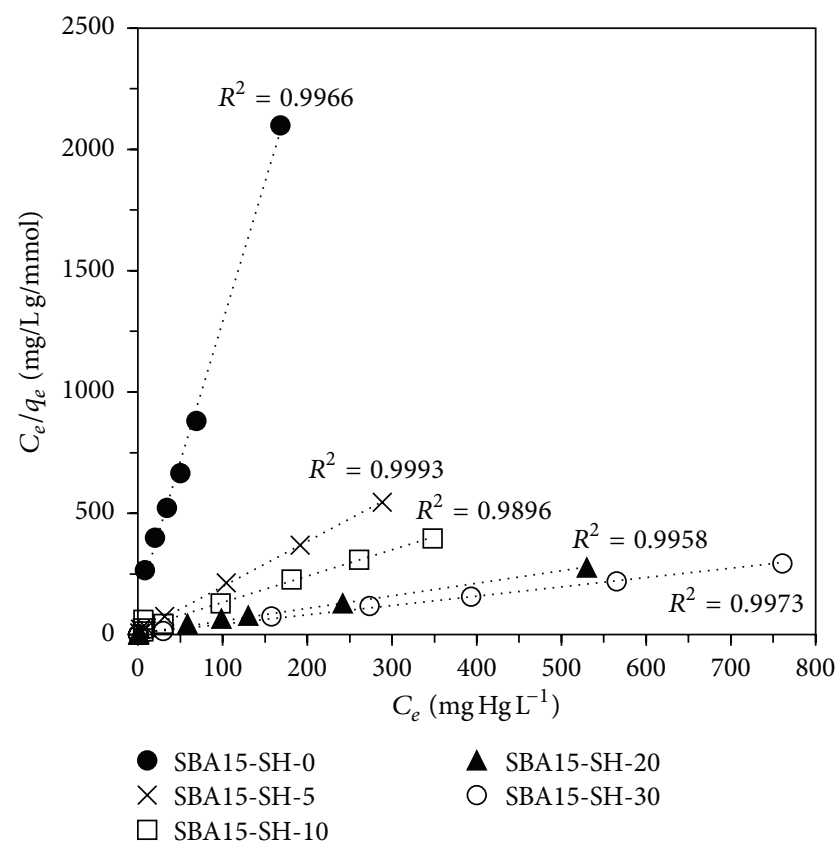

(b)

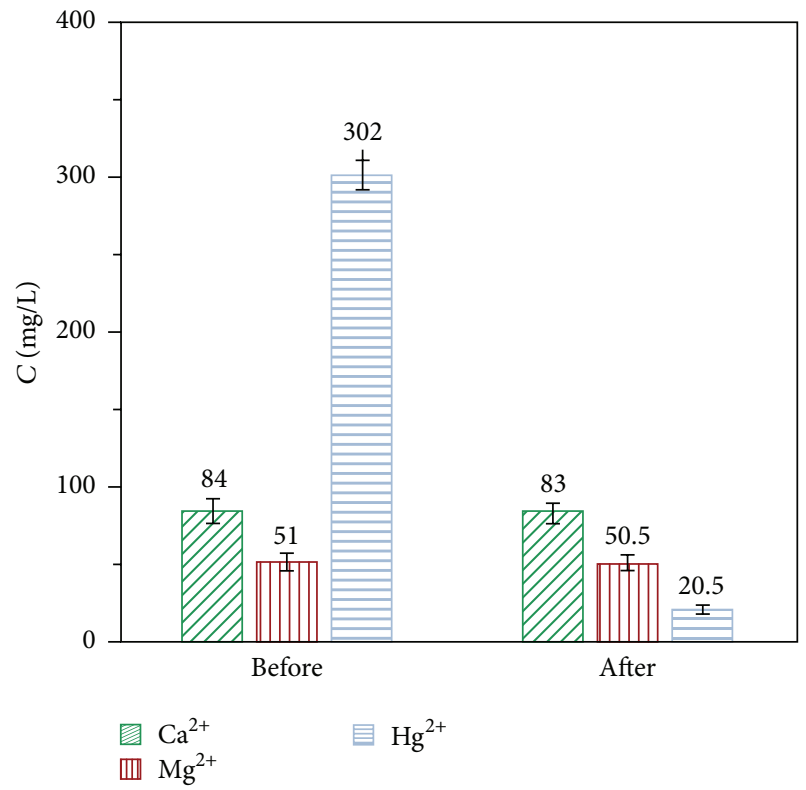

(d)

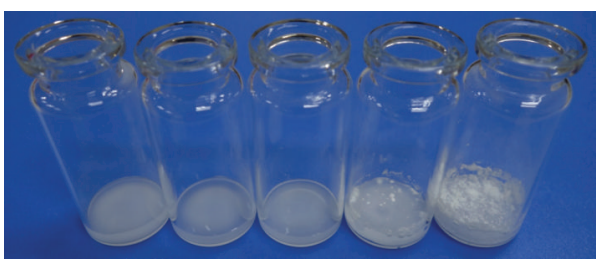

SBA15- SBA15- SBA15- SBA15- SBA15-

SH-0 SH-5 SH-10 SH-20 SH-30

(e)

FIGURE 4: Evaluation of mercury adsorption ability of the materials. (a) Mercury adsorption isotherms (obtained at $20^{\circ} \mathrm{C}$ and $\mathrm{pH}=1.2$ ) and (b) Langmuir linear plots of all materials. (c) The influence of $\mathrm{pH}$ on the mercury adsorption of SBA15-SH-10. (d) Competitive adsorption experiments in vitro were tested in aqueous system in the presence of SBA15-SH-10 and a mixture of $\mathrm{Hg}$ (II), $\mathrm{Ca}$ (II), and $\mathrm{Mg}$ (II). The results are indicated as the concentration of the metal ions before and after the adsorption; (e) shows the water dispersibility of the materials. 


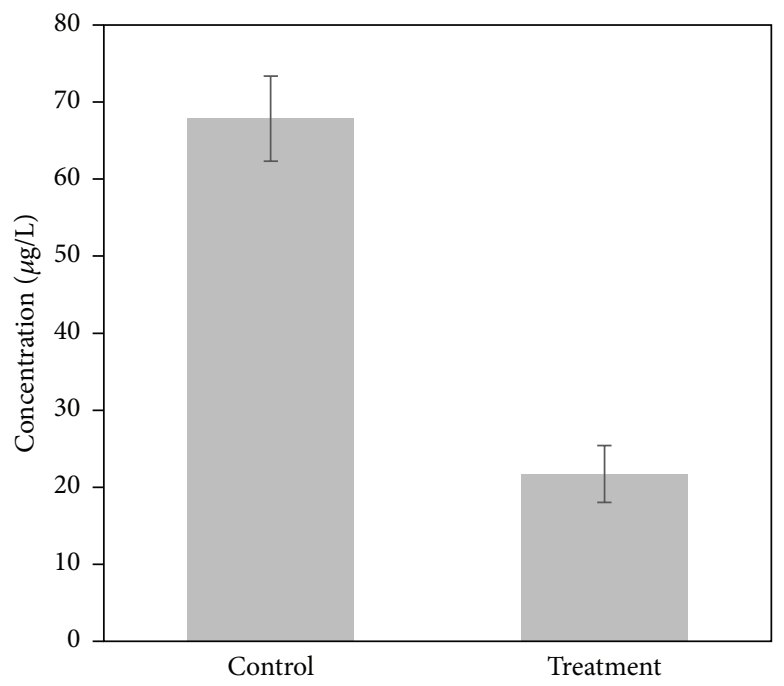

(a)

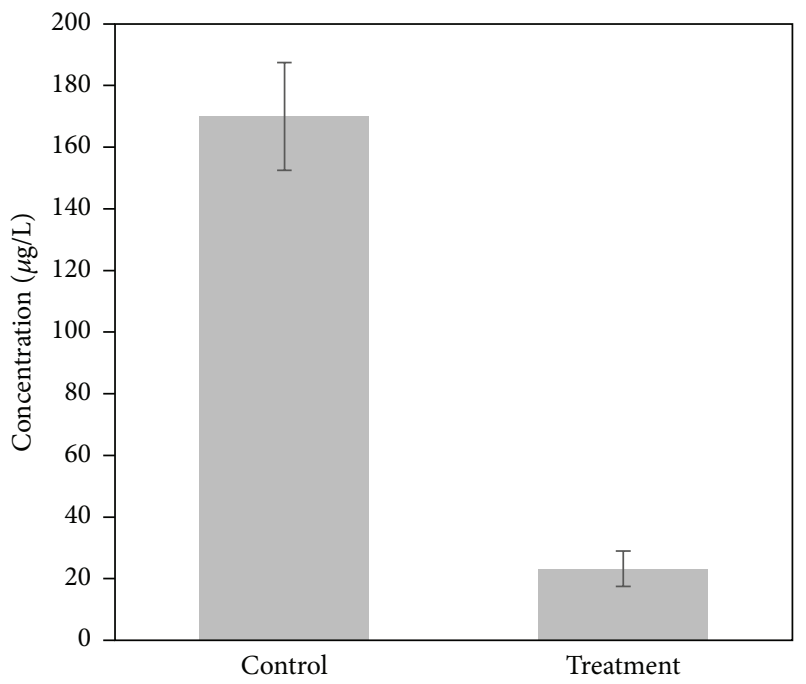

(b)

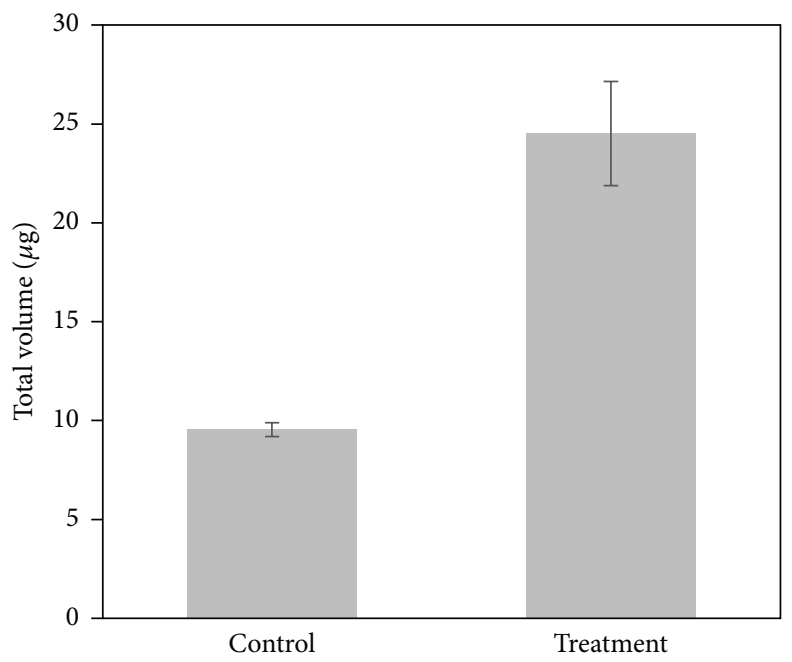

(c)

FIGURE 5: Mercury levels of (a) plasma, (b) urine, and (c) faeces of control group and treatment group.

because the SBA15-SH-10 has a strong adsorption capacity for mercury (II) and the mercury (II) loaded on material could be excluded with the faeces.

Mercury (II) can be absorbed by gastrointestinal tract as soon as ingestion and directly cause local trauma due to the salts corrosive nature $[20,21]$. Simultaneously, it can react with biochemical thiol of cysteine or glutathione, leading to the inactivity of protease $[27,28]$. In clinics, gastrolavage and clyster are commonly used to remove the toxin. Even though patients receive the treatment, toxin remnant is possible. Furthermore, patients often receive no efficient treatment before hospitalization and available chelating agents are used to eliminate heavy metal in blood $[13,14]$. Therefore, in order to solve this problem, we designed this oral agent for mercury detoxification. This agent can selectively capture mercury (II) and provide a shelter for the adsorbed mercury preventing further chemical reaction. Previous studies also prove that thiol-functionalized materials have high affinity for heavy metal and can effectively trap them $[29,30]$. Our in vivo data shows that the thiol-functionalized mesoporous silica administrated through gastrointestinal route can significantly decrease the mercury levels of blood and urine. Hence, the thiol-functionalized mesoporous silica has potential application in the prevention of heavy metal poisoning.

\section{Conclusions}

Here, we prepared thiol-functionalized mesoporous silica (SBA15-SH-10) as an oral formulation for the prevention and treatment of heavy metal poisoning. The SBA-SH-10 exhibits uniform mesopores and high water dispersibility. In simulated gastrointestinal fluid, thiol-functionalized mesoporous silica can selectively capture heavy metal, showing a very high affinity for inorganic mercury (II). In vivo, the material can significantly decrease the blood and urine mercury levels of the acute mercury poisoning rats. Hence, thiolfunctionalized mesoporous silica has potential application in preventing and treating heavy metal poisoning. 


\section{Competing Interests}

The authors declare that they have no competing interests.

\section{Authors' Contributions}

Wei Zhao and Bo Song contributed equally to this work.

\section{Acknowledgments}

This work was supported by the National Natural Science Foundation (81422025, 81301907, and 81572990), 863 Program (2014AA020509), and the Foundation for Distinguished Young Scientists of Sichuan Province (2016JQ0020).

\section{References}

[1] A. Burns, H. Ow, and U. Wiesner, "Fluorescent core-shell silica nanoparticles: towards 'lab on a particle' architectures for nanobiotechnology," Chemical Society Reviews, vol. 35, no. 11, pp. 1028-1042, 2006.

[2] Y. Piao, A. Burns, J. Kim, U. Wiesner, and T. Hyeon, "Designed fabrication of silica-based nanostructured particle systems for nanomedicine applications," Advanced Functional Materials, vol. 18, no. 23, pp. 3745-3758, 2008.

[3] B. G. Trewyn, I. I. Slowing, S. Giri, H.-T. Chen, and V. S.-Y. Lin, "Synthesis and functionalization of a mesoporous silica nanoparticle based on the sol-gel process and applications in controlled release," Accounts of Chemical Research, vol. 40, no. 9, pp. 846-853, 2007.

[4] M. Vallet-Regí, F. Balas, and D. Arcos, "Mesoporous materials for drug delivery," Angewandte Chemie-International Edition, vol. 46, no. 40, pp. 7548-7558, 2007.

[5] A. Popat, S. B. Hartono, F. Stahr, J. Liu, S. Z. Qiao, and G. Q. (Max) Lu, "Mesoporous silica nanoparticles for bioadsorption, enzyme immobilisation, and delivery carriers," Nanoscale, vol. 3, no. 7, pp. 2801-2818, 2011.

[6] S.-H. Wu, Y. Hung, and C.-Y. Mou, "Mesoporous silica nanoparticles as nanocarriers," Chemical Communications, vol. 47, no. 36, pp. 9972-9985, 2011.

[7] I. I. Slowing, J. L. Vivero-Escoto, C.-W. Wu, and V. S.-Y. Lin, "Mesoporous silica nanoparticles as controlled release drug delivery and gene transfection carriers," Advanced Drug Delivery Reviews, vol. 60, no. 11, pp. 1278-1288, 2008.

[8] E. G. Pacyna, J. M. Pacyna, K. Sundseth et al., "Global emission of mercury to the atmosphere from anthropogenic sources in 2005 and projections to 2020," Atmospheric Environment, vol. 44, no. 20, pp. 2487-2499, 2010.

[9] D. G. Streets, Q. Zhang, and Y. Wu, "Projections of global mercury emissions in 2050," Environmental Science and Technology, vol. 43, no. 8, pp. 2983-2988, 2009.

[10] D. R. Baldwin and W. J. Marshall, "Heavy metal poisoning and its laboratory investigation," Annals of Clinical Biochemistry, vol. 36, no. 3, pp. 267-300, 1999.

[11] M. L. Gou, X. Qu, W. Zhu et al., "Bio-inspired detoxification using 3D-printed hydrogel nanocomposites," Nature Communications, vol. 5, article 3374, 2014.

[12] F. Xu, T. Y. Kang, J. Deng et al., "Functional nanoparticles activate a decellularized liver scaffold for blood detoxification," Small, vol. 12, no. 15, pp. 2067-2076, 2016.
[13] A. L. Miller, "Dimercaptosuccinic acid (DMSA), a non-toxic, water-soluble treatment for heavy metal toxicity," Alternative Medicine Review, vol. 3, no. 3, pp. 199-207, 1998.

[14] H. V. Aposhian, R. M. Maiorino, D. Gonzalez-Ramirez et al., "Mobilization of heavy metals by newer, therapeutically useful chelating agents," Toxicology, vol. 97, no. 1-3, pp. 23-38, 1995.

[15] S. J. L. Billinge, E. J. McKimmy, M. Shatnawi et al., "Mercury binding sites in thiol-functionalized mesostructured silica," Journal of the American Chemical Society, vol. 127, no. 23, pp. 8492-8498, 2005.

[16] X. Feng, G. E. Fryxell, L.-Q. Wang, A. Y. Kim, J. Liu, and K. M. Kemner, "Functionalized monolayers on ordered mesoporous supports," Science, vol. 276, no. 5314, pp. 923-926, 1997.

[17] T. Zhou, I. Georgiev, X. Wu et al., "Structural basis for broad and potent neutralization of HIV-1 by antibody VRC01," Science, vol. 329, no. 5993, pp. 811-817, 2010.

[18] T. Sangvanich, J. Morry, C. Fox et al., "Novel oral detoxification of mercury, cadmium, and lead with thiol-modified nanoporous silica," ACS Applied Materials and Interfaces, vol. 6, no. 8, pp. 5483-5493, 2014.

[19] D. Zhao, J. L. Feng, Q. S. Huo et al., “Triblock copolymer syntheses of mesoporous silica with periodic 50 to 300 angstrom pores," Science, vol. 279, no. 5350, pp. 548-552, 1998.

[20] A. S. M. Chong and X. S. Zhao, "Functionalization of SBA-15 with APTES and characterization of functionalized materials," The Journal of Physical Chemistry B, vol. 107, no. 46, pp. 1265012657, 2003.

[21] P. D. Yang, D. Y. Zhao, D. I. Margolese, B. F. Chmelka, and G. D. Stucky, "Generalized syntheses of large-pore mesoporous metal oxides with semicrystalline frameworks," Nature, vol. 396, no. 6707, pp. 152-155, 1998.

[22] S. Y. Choi, M. Mamak, N. Coombs, N. Chopra, and G. A. Ozin, "Thermally stable two-dimensional hexagonal mesoporous nanocrystalline anatase, meso-nc- $\mathrm{TiO}_{2}$ : bulk and crackfree thin film morphologies," Advanced Functional Materials, vol. 14, no. 4, pp. 335-344, 2004.

[23] Y. Li, W. Xiong, C. Wang, B. Song, and G. Zhang, "Synthesis of hexagonal mesoporous silicates functionalized with amino groups in the pore channels by a co-condensation approach," RSC Advances, vol. 6, no. 59, pp. 53991-54000, 2016.

[24] K. S. W. Sing, D. H. Everett, R. A. W. Haul et al., "Reporting physisorption data for gas/solid systems with special reference to the determination of surface area and porosity (Recommendations 1984)," Pure and Applied Chemistry, vol. 57, no. 4, pp. 603-619, 1985.

[25] M. Kruk and M. Jaroniec, "Gas adsorption characterization of ordered organic-inorganic nanocomposite materials," Chemistry of Materials, vol. 13, no. 10, pp. 3169-3183, 2001.

[26] R. Sanz, G. Calleja, A. Arencibia, and E. S. Sanz-Pérez, "Amino functionalized mesostructured SBA-15 silica for $\mathrm{CO}_{2}$ capture: exploring the relation between the adsorption capacity and the distribution of amino groups by TEM," Microporous and Mesoporous Materials, vol. 158, pp. 309-317, 2012.

[27] T. W. Clarkson, “The three modern faces of mercury," Environmental Health Perspectives, vol. 110, supplement 1, pp. 11-23, 2002.

[28] T. W. Clarkson, J. B. Vyas, and N. Ballatori, "Mechanisms of mercury disposition in the body," American Journal of Industrial Medicine, vol. 50, no. 10, pp. 757-764, 2007. 
[29] R. A. Goyer, M. G. Cherian, M. M. Jones, and J. R. Reigart, "Role of chelating agents for prevention, intervention, and treatment of exposures to toxic metals," Environmental Health Perspectives, vol. 103, no. 11, pp. 1048-1052, 1995.

[30] W. Yantasee, C. L. Warner, T. Sangvanich et al., "Removal of heavy metals from aqueous systems with thiol functionalized superparamagnetic nanoparticles," Environmental Science and Technology, vol. 41, no. 14, pp. 5114-5119, 2007. 

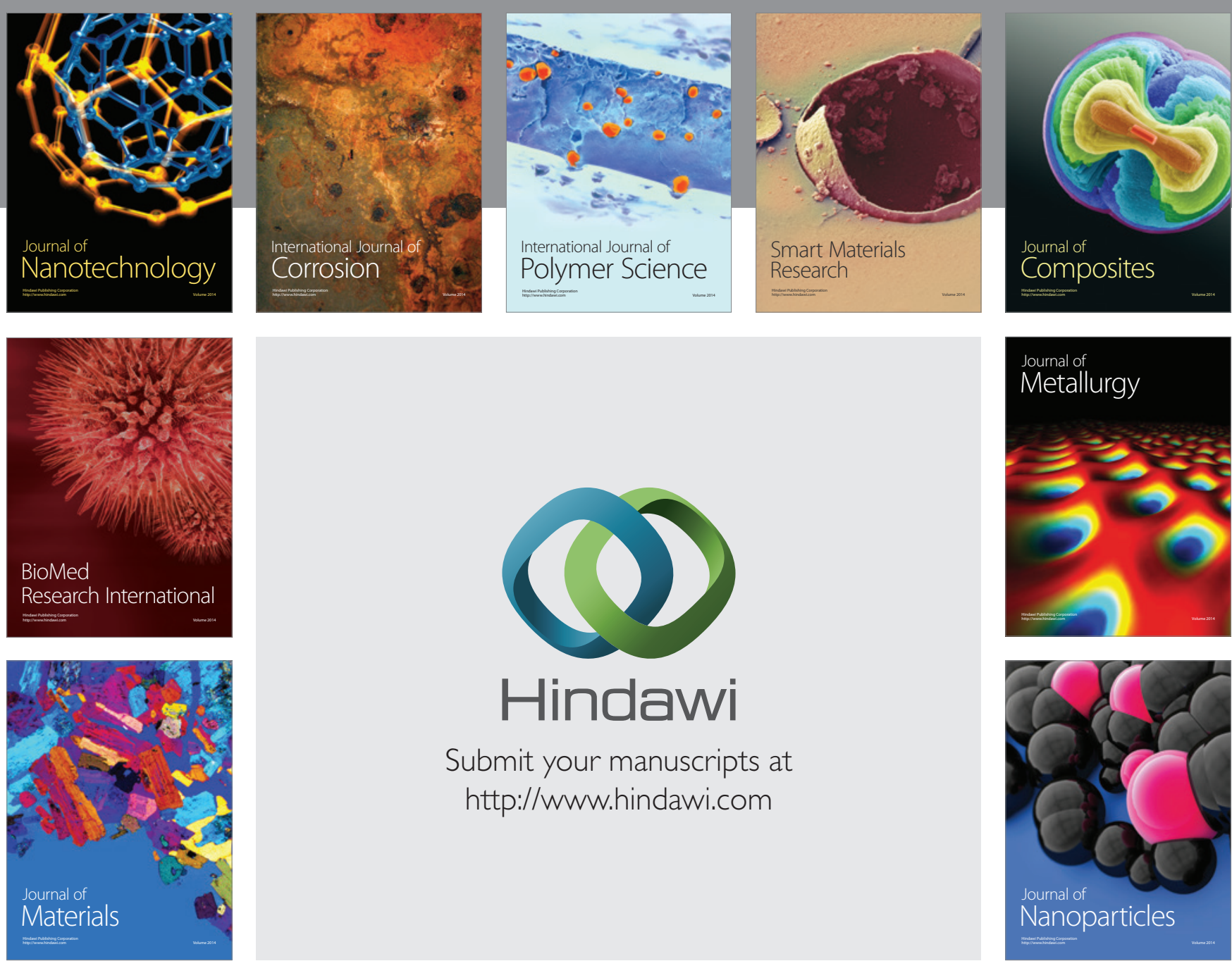

\section{Hindawi}

Submit your manuscripts at

http://www.hindawi.com

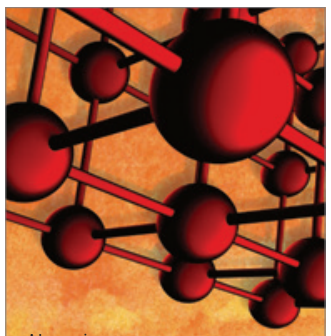

Materials Science and Engineering
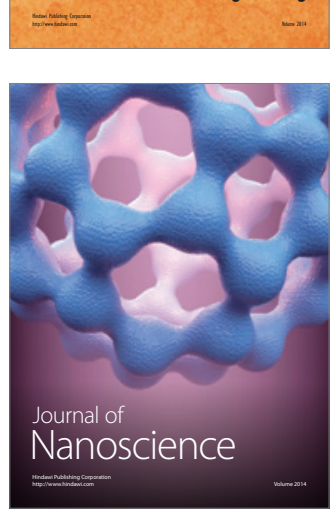
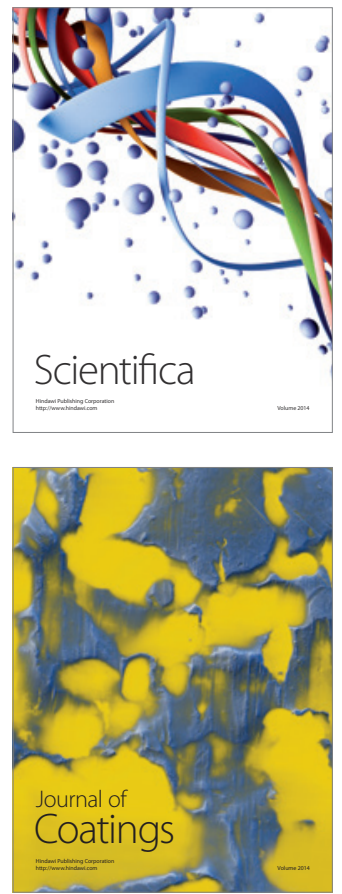
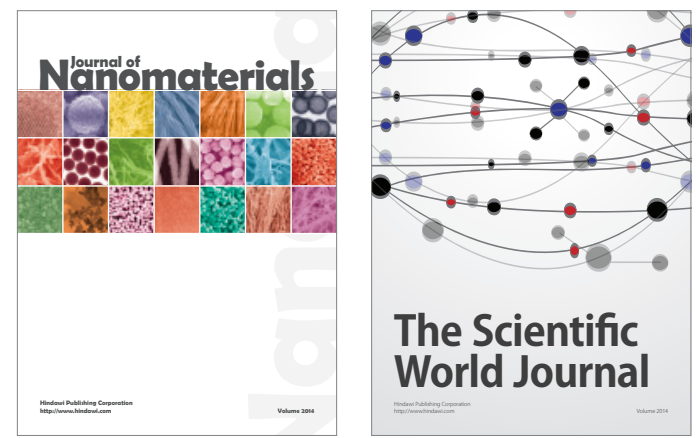

The Scientific World Journal

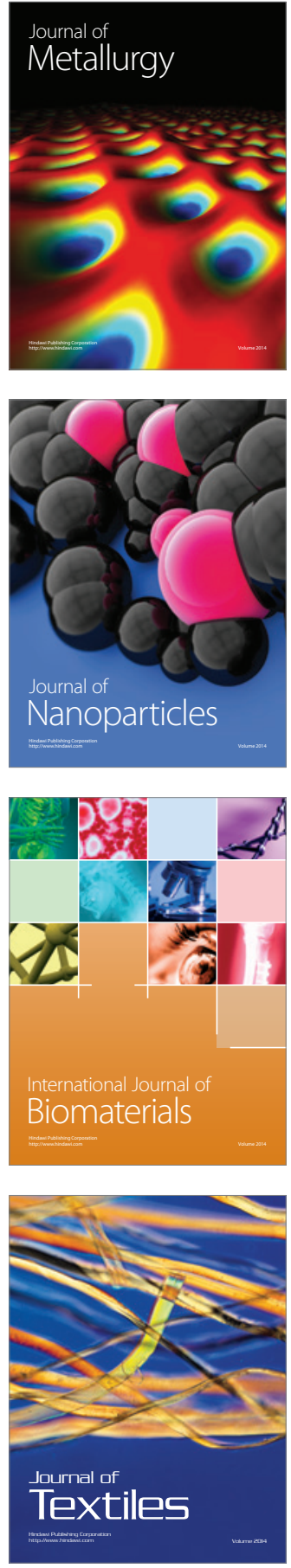